\title{
FGFR4 Links Glucose Metabolism and Chemotherapy Resistance in Breast Cancer
}

\author{
Min Xu $u^{a, b}$ Shuzheng Chen ${ }^{c}$ Weibin Yang ${ }^{a, b} \quad$ Xue Cheng ${ }^{a, b} \quad$ Yani Ye $e^{a, b}$ \\ Jianting Mao ${ }^{\mathrm{a}, \mathrm{b}} \quad$ Xulu Wu $\mathrm{Wu}^{\mathrm{a}, \mathrm{b}}$ Li Huang ${ }^{\mathrm{d}}$ Jiansong Jia,b
}

aKey Laboratory of Imaging Diagnosis and Minimally Invasive Intervention Research, bepartments of Radiology, 'Breast Surgery, The Fifth Affiliated Hospital of Wenzhou Medical University, Affiliated Lishui Hospital of Zhejiang University, The Central Hospital of Zhejiang, Lishui, Zhejiang, dSchool of Materials Science and Engineering, Shanghai Key Laboratory of D\&A for Metal-Functional Materials, Tongji University, Shanghai, PR China

\section{Key Words}

Fgf receptor 4 • Glycolytic flux • Chemoresistance • Breast cancer

\begin{abstract}
Background/Aims: Poor response to chemotherapy leads to the relapse and metastatic progression of tumors. Reprogrammed glucose metabolism is one of the important hallmarks of cancer that facilitates cancer cell survival, proliferation and chemoresistance. However, the precise fate of glucose metabolism and its role in therapy responsiveness in cancers remains largely unexplored. Methods: The glycolytic phenotype of doxorubicin (ADR)-resistant breast cancer cells and their parental cells was assessed by measuring glucose uptake, lactate release, and extracellular acidification rate (ECAR). Protein expression was detected by Western blotting analysis and mRNA expression was detected using q-PCR. Cell survival ratio was determined by the cell counting kit 8 assay. The role of fibroblast growth factor receptor 4 (FGFR4) in glycolysis, chemoresistance, and the underlying mechanisms were studied by using gene expression microarray and short hairpin RNA-mediated gene knockdown. Results: We found that glycolytic flux are increased in two doxorubicin (ADR)-resistant breast cancer cell lines compared with their parental wild type cells, as demonstrated by increased glucose uptake, lactate release, and extracellular acidification rate (ECAR). By gene expression microarray, we identified FGFR4 as a critical modulator of ADR resistance and enhanced glucose metabolism. Genetic silencing of FGFR4 increased the chemosensitivity and suppressed the enhanced glycolytic flux in ADR-resistant cells. Mechanistically, activation of FGFR4 signaling in ADRresistant cells led to the phosphorylation of FGF receptor substrate 2 (FRS2) and further activated the downstream MAPK/ERK signaling. Pharmacological inhibition of FGFR4-FRS2ERK signaling pathway significantly blocked the chemoresistant and glycolytic phenotypes of ADR-resistant cells. Conclusion: Our findings suggest that high levels of FGFR4 can increase glucose metabolism and lead to chemoresistance in breast cancer and reveal the mechanistic basis for targeting FGFR4 as a therapeutic opportunity for chemoresistant tumors.
\end{abstract}

M. Xu, S. Chen and W. Yang contributed equally to this work. 


\section{Cellular Physiology Cell Physiol Biochem 2018;47:151-160

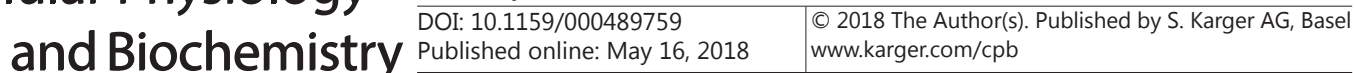 \\ Xu et al.: FGFR4 Correlates the Glycolytic and Chemoresistant Phenotypes in Breast}

Cancer

\section{Introduction}

Breast cancer is the major cause of cancer-related mortality in women. Despite the development of early clinical detection methods and breast cancer therapy, some patients with advanced breast cancer have recurrent disease [1,2]. Although personalized therapy is popular, for patients with advanced breast cancer, chemotherapy is still considered the alternative treatment option [1]. However, chemoresistance is the most common and critical limitation of breast cancer treatment. Doxorubicin (ADR), a derivative of the natural product daunomycin, works by intercalating DNA and inhibiting the religation step of topoisomerase II. It has long been used as a first-line chemotherapeutic drug to treat breast cancer [3-5]. Unfortunately, doxorubicin drug resistance appears in nearly 50\% of treated patients [3]. The development of chemoresistance in breast cancer can be either intrinsic or acquired. Several molecular mechanisms are associated with the chemoresistant phenotype of breast cancer. It is widely recognized that up-regulation of the drug transporter family known as the adenosine triphosphate-binding cassette (ABC) drug transporter family contributes to drug resistance by regulating drug efflux. Moreover, cancer stem cells (CSC), changes of anticancer drug targets, and dysregulation of key oncogenic signaling pathways also play vital roles in the chemoresistant phenotype of breast cancer [1]. Recent studies have revealed the profound effects of glucose metabolism on the development of chemoresistance $[3,6]$.

A hallmark of cancer is the reprogramming of energy metabolism to promote cell survival and inhibit cell apoptosis [6-8]. A primary metabolic alteration associated with cellular transformation is enhanced aerobic glycolysis, also known as the "Warburg effect". The Warburg Effect is defined as an increase in the rate of glucose uptake and the fermentation of glucose to lactate, even in the presence of oxygen [9]. The Warburg Effect not only contributes to rapid ATP synthesis but also supports the biosynthetic requirements of uncontrolled proliferation $[10,11]$. However, the precise fate of glucose metabolism and its role in therapy responsiveness in cancers remains largely unexplored.

The fibroblast growth factor (FGFs)-FGFR signaling system has been implicated in diverse biological processes during embryo development, wound healing, and angiogenesis $[12,13]$. The FGFR protein family consists of four highly conserved receptor tyrosine kinase members (FGFR1, 2, 3 and 4). Activation of FGFRs leads to receptor dimerization, autophosphorylation and activation of downstream signaling pathways governing various oncogenic processes, including cell differentiation, proliferation, metastasis, angiogenesis and chemoresistance $[14,15]$. The overexpression of FGFR4 has been reported in many cancers, and high levels of FGFR4 expression are associated with poor survival in cancer patients [16-20].

In this study, we first present a mechanism of ADR chemoresistance by which breast cancer cells increase glycolytic flux. Subsequently, expression profile microarray analysis and a loss-of-function study revealed that FGFR4 is a critical modulator of ADR chemoresistance and enhanced glycolytic flux in ADR-resistant cells. Finally, pharmacological inhibition of FGFR4-FRS2-ERK signaling blocked the chemoresistant and glycolytic phenotypes of ADRresistant cells.

\section{Materials and Methods}

\section{Cell culture and reagents}

The human breast cancer cell lines MCF-7 and MDA-MB-231 were purchased from the American Type Culture Collection (Manassas, VA, USA). The MCF-7/ADR cell line (ADR-resistant estrogen receptor positive human breast adenocarcinoma) was obtained from the Institute of Biochemistry and Cell Biology, Chinese Academy of Science (Shanghai, China). TogenerateMBA-MD-231/ADR cells, MBA-MD-231 cells were chronically incubated with increased concentrations of doxorubicin for 12 months. On average, the concentration of doxorubicin was increased every 2 weeks. The resistance of the ADR-R cells to ADR was confirmed by measuring the $\mathrm{IC}_{50}$ value using the CCK-8 assay. The cells were grown in Dulbecco's modified Eagle's medium (Gibco, MD, USA) containing 10\% fetal bovine serum (FBS) and 100U/ml penicillin/ 
streptomycin in a humidified atmosphere containing $5 \% \mathrm{CO}_{2}$ at $37^{\circ} \mathrm{C}$. ADR $(2 \mu \mathrm{mol} / \mathrm{L})$ was added to the culture medium of the MBA-MD-231/ADR and MCF-7/ADR cells to maintain the ADR resistance. ADR-HCl and DMSO were purchased from Sigma-Aldrich (Shanghai, China).The inhibitors PD173074 and U0126 were purchased from Selleck (Shanghai, China).

\section{Measurement of glucose uptake and lactate release}

Culture supernatants were collected for the measurement of glucose uptake and lactate release. The lactate concentration in the supernatant was determined utilizing Hexokinase Kits (Sigma, USA) and Lactate Assay Kits (Sigma \#MAK064) according to the manufacturer's instructions. All the raw data were normalized by cell density measured by the crystal violet method.

\section{Measurement of extracellular acidification rates (ECARs)}

Extracellular acidification rates (ECARs) were determined using the Seahorse Extracellular Flux (XFe96) analyzer (Seahorse Bioscience, USA). Cells were seeded at a density of $1 \times 10^{4}$ per well in XFe96 cell culture plates and incubated for $16 \mathrm{~h}$ in $\mathrm{F}$ medium at $37^{\circ} \mathrm{C}$ in a $5 \% \mathrm{CO}_{2}$ humidified atmosphere. Subsequently, cells were washed in XF assay medium and kept in XF assay medium at $37^{\circ} \mathrm{C}$ in a non- $\mathrm{CO}_{2}$ incubator for 1 hr. Then, glucose, oligomycin, and 2-DG in XF assay medium were loaded into the injection ports in the XFe96 sensor cartridge to determine the ECARs. The experiment was performed with 6 replicates in each experiment.

\section{Real-time quantitative PCR}

Total RNA was extracted using TRIzol reagent (Invitrogen, USA) according to the manufacturer's protocol. cDNA was synthesized using the Superscript III First Strand Synthesis Kit (Invitrogen, USA).Quantitative RT-PCR was performed using SYBR green assays (Invitrogen, USA) and an Applied Biosystems 7500. The gene-specific primers are shown in Table 1. The relative expression of each mRNA was calculated using the equation $2^{\wedge-\Delta \Delta C \mathrm{t}}$. All PCR assays were performed in triplicate.

\section{Gene expression microarrays}

Sample preparation and microarray hybridizations and assays were performed by the Biotechnology Corporation (Shanghai, China). Briefly, RNA was purified using mRNA Isolation Kits (Ambion, Austin, TX) according to the manufacture's protocol. The cDNA waslabeled and then hybridized to anAgilent Human SurePrint G3 Human GE 8 × 60 k mRNA microarray chip (Agilent Technologies). To select differentially expressed genes, we used threshold values of $\geq 2$ and $\leq-2$-fold changes and a Benjamini-Hochberg corrected $p$ value $<0.05$ (multiple testing, Benjamini-Hochberg method).

\section{siRNA transfection}

Specific siRNAs targeting FGFR4 and the controls were purchased from GenePharma (Shanghai, China). For siRNA transfection, cells were seeded in 6-well culture plates and transfected with $50 \mathrm{nM}$ siRNA using Lipofectamine 2000 (Invitrogen, Carlsbad, CA, USA) according to the manufacturer's instructions. Then, $48 \mathrm{~h}$ after transfection, the cells were analyzed by Western blotting and real-time quantitative PCR.
Table 1. The sequences of primers used in this study

\begin{tabular}{lcc}
\hline Gene & Forward primer (5'-3') & Reverse primer (5'-3') \\
\hline GLUT1 & TCTGGCATCAACGCTGTCTTC & CGATACCGGAGCCAATGGT \\
HK2 & GAGCCACCACTCACCCTACT & CCAGGCATTCGGCAATGTG \\
PFK & GCTGGGCGGCACTATCATT & TCAGGTGCGAGTAGGTCCG \\
ALDOA & CAGGGACAAATGGCGAGACTA & GGGGTGTGTTCCCAATCTT \\
ENO1 & AAAGCTGGTGCCGTTGAGAA & GGTTGTGGTAAACCTCTGCTC \\
PKM2 & ATGTCGAAGCCCCATAGTGAA & TGGGTGGTGAATCAATGTCCA \\
LDHA & TTGACCTACGTGGCTTGGAAG & GGTAACGGAATCGGGCTGAAT \\
PDK1 & GAGAGCCACTATGGAACACCA & GGAGGTCTCAACACGAGGT \\
TFDP3 & CAGCATCTCCGACGACAAATC & ACGTGCCATTAGACCTGGAAC \\
LOXL2 & CTCCTCCTACGGCAAGGGA & ATGTCCTCCACCTGGATATTCA \\
AQP3 & GGGGAGATGCTCCACATCC & AAAGGCCAGGTTGATGGTGAG \\
OCT4 & CTTGAATCCCGAATGGAAAGGG & GTGTATATCCCAGGGTGATCCTC \\
ABCC5 & AGTCCTGGGTATAGAAGTGTGAG & ATTCCAACGGTCGAGTTCTCC \\
PMP22 & GATCCTGTCGATCATCTTCAGC & AGCACTCATCACGCACAGAC \\
PAK1 & CAACTCGGGACGTGGCTAC & CAGTATTCCGGGTCAAAGCAT \\
HPIP & TGGGCAGAGAGACCGGAAG & TCATGGCTGTCCTTAGTCCCT \\
FGFR4 & GAGGGGCCGCCTAGAGATT & CAGGACGATCATGGAGCCT \\
NRF2 & TCAGCGACGGAAAGAGTATGA & CCACTGGTTTCTGACTGGATGT \\
\hline
\end{tabular}


$\mathrm{Xu}$ et al.: FGFR4 Correlates the Glycolytic and Chemoresistant Phenotypes in Breast Cancer

Cell viability assay

Cell viability was measured using a Cell Counting Kit 8 (CCK-8, Dojindo, Japan). Cells were plated in 96well plates at a density of $5 \times 10^{3}$ cells/well and incubated for $24 \mathrm{~h}$. Cells were cultured for an additional 48 $\mathrm{h}$ with the indicated treatment. Then, $10 \mu \mathrm{l}$ of the CCK- 8 reagent was added to each well, and the cells were incubated for $1 \mathrm{~h}$ at $37^{\circ} \mathrm{C}$. The absorbance in each well was measured at $450 \mathrm{~nm}$ using a multifunctional microplate reader (Bio-TEK, USA). The data from 3 independent experiments were analyzed.

\section{Western blotting}

Cultured cells were washed with PBS and lysed with RIPA lysis buffer (Millipore, Billerica, MA) supplemented with complete EDTA-free protease inhibitor cocktail (Roche, Indianapolis, IN)and phosphatase inhibitor cocktails 1 and 2 (Sigma-Aldrich, St. Louis, MO). Protein concentrations were quantified using a Bio-Rad DC protein assay kit (Bio-Rad, USA). Samples $(15 \mu \mathrm{g}$ ) of total cell lysates were separated on $10 \%$ sodium dodecyl sulfate-polyacrylamide (SDS) gels and electrotransferred to polyvinylidene difluoride (PVDF) membranes. The membranes were blocked by a1-hour incubation with 5\% nonfat dry milk in Trisbuffered saline with $1 \%$ Tween. After being blocked, the membranes were incubated with specific primary antibodies against the selected proteins at $4^{\circ} \mathrm{C}$ overnight. Then, the membranes were incubated with the species-matched peroxidase-conjugated secondary antibody. Specific reactive proteins were detected by enhanced chemiluminescence (Pierce). The following commercial antibodies were used for Western blot analysis: anti-FGFR4 (1:500, Abcam, ab5481), anti-phospho-FRS2 $\alpha$ (1:1000, Abcam, ab193363), anti-FRS2 $\alpha$ (1:1000, Abcam, ab10425), anti-phospho-Akt (1:1000, CST, \#4060), anti-Akt (1:1000, CST, \#4691), antiphospho-Erk1/2 (1:1000, CST, \#9101), anti-Erk1/2 (1:1000, CST, \#4695), anti-phospho-NF-кB (1:1000, CST, \#3039), anti-NF-кB (1:1000, CST, \#6956), and anti-GAPDH antibody (1:2000; Abcam, ab8245).

\section{Statistical analysis}

All values are expressed as the mean \pm SD of at least 3 individual experiments. The statistical comparisons between the groups were performed using one-way analysis of the variance (ANOVA) or Student's t-test. Statistical analyses were performed using SPSS 13.0 statistical package software (SPSS, Chicago, IL, USA) or GraphPad Prism (GraphPad Software Inc., San Diego, CA). P values less than 0.05 were considered statistically significant $\left({ }^{*} \mathrm{p}<0.05,{ }^{* *} \mathrm{p}<0.01,{ }^{* * *} \mathrm{p}<0.001\right)$.

\section{Results}

\section{ADR-resistant breast cancer cells exhibit increased glucose metabolism}

To investigate the metabolic alterations associated with ADR resistance, two breast cancer cell models with acquired ADR resistance, MCF7/ADR and MDA-MB-231/ADR, were used. Compared with WT cells, ADR-resistant cells had significantly higher IC $_{50}$ values (Fig. 1A).To compare the glucose metabolism of ADR-R cells with that of the WT cells, we first assayed glucose uptake after culturing the cells for $24 \mathrm{hr}$. We found that MCF7/ADR-R and MDA-MB-231/ADR-R cells displayed significantly increased glucose uptake compared with the WT cells (Fig. 1B). Consistently, ADR-R cells also demonstrated more lactate production than WT cells (Fig. 1C). To confirm the correlation between ADR resistance and glucose metabolism, we measured the extracellular acidification rates (ECARs) using the XFe96 extracellular flux analyzer. As shown in Fig. 1D, ADR-R cells demonstrated increased extracellular acidification rates compared to their parental WT cells. In addition, by measuring the expression of glycolytic enzymes, we observed that the expression of genes involved in glucose metabolism was also increased in ADR-R cells (Fig. 1E). In summary, these findings suggest that glucose metabolism is enhanced in ADR-resistant cells.

Inhibiting glycolysis suppresses the chemoresistant phenotype of breast cancer cells

To determine the dependence of the ADR resistance phenotype on glucose metabolism, we cultured the ADR-R cells and their parental WT cells in low glucose conditions (1 mM glucose) for $24 \mathrm{hr}$. As shown in Fig. 2A and 2B, ADR-R cells displayed a significant response to ADR treatment in both ADR-R cell lines. To confirm this observation, we inhibited glycoly- 
$\mathrm{Xu}$ et al.: FGFR4 Correlates the Glycolytic and Chemoresistant Phenotypes in Breast Cancer

Fig. 1. ADR-resistant breast cancer cells exhibit increased glucose metabolism. (A) Cells were treated with the indicated concentrations of ADR. To assess cell survival, CCK-8 assays were performed $48 \mathrm{hr}$ later. (B) The relative glucose consumption of ADR-R and WT cells as determined by colorimetric analysis $(n=5)$. (C) The relative lactate production of ADR-R and WT cells as determined by colorimetric analysis (n = 5). (D) WT and ADR-R breast cancer cells were seeded at a density of $1 \times$ $10^{4}$ per well in 96-well plates and exposed to glucose, oligomycin, and 2-DG to measure the extracellular acidification rate (ECAR). (E) Real-time qPCR analysis for glycolytic genes in WT and ADR-R breast cancer cells. ${ }^{*} \mathrm{p}<0.05$; ${ }^{* *} \mathrm{p}<0.01 ;{ }^{* * *} \mathrm{p}<0.001$.

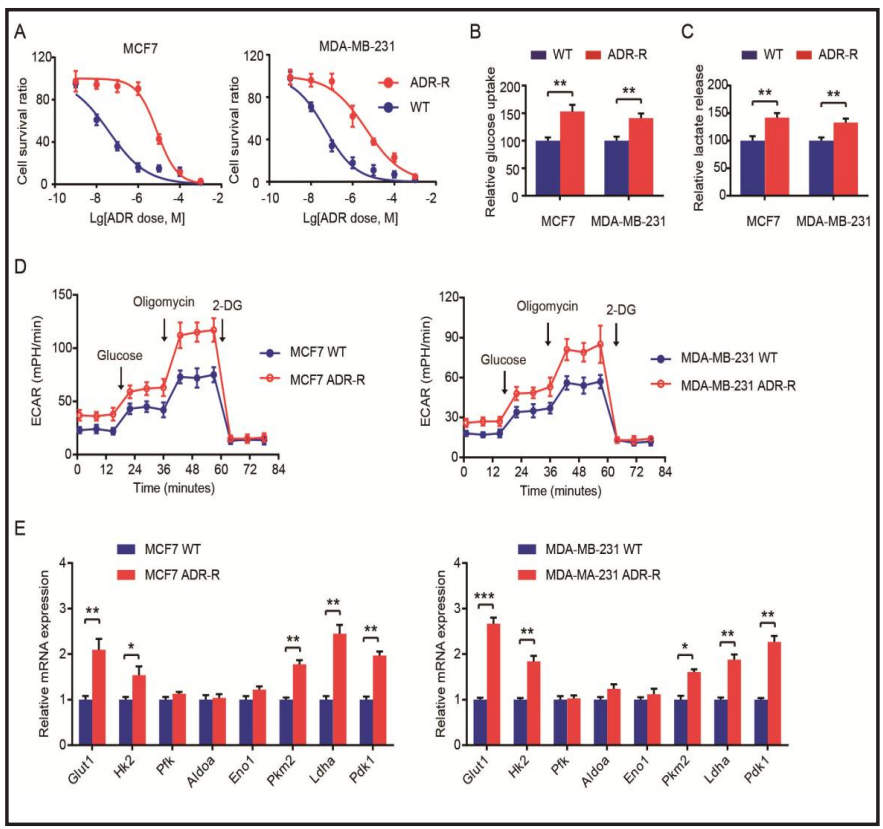

Fig. 2. Inhibiting glycolysis suppresses the chemoresistant phenotype of breast cancer cells. (A-B)The relative chemosensitivity of MCF7 ADR-R (A) and MDA-MB-231 ADR-R (B) cells in culture medium containing $1 \mathrm{mM}$ glucose. Cells were treated with $1 \mu \mathrm{M}$ ADR for $24 \mathrm{hr}$, and their survival was evaluated by CCK-8 analysis. (C-D)The relative chemosensitivities of MCF7 ADR-R (C) and MDA-MB-231 ADR-R (D) cells in the presence of $20 \mathrm{mM}$ 2-DG. Cells were treated with $1 \mu \mathrm{M}$ ADR for $24 \mathrm{hr}$, and their viability was determined byCCK-8 analysis. ${ }^{*} \mathrm{p}<0.05 ;{ }^{* *} \mathrm{p}<0.01$; $* * * \mathrm{p}<0.001$.

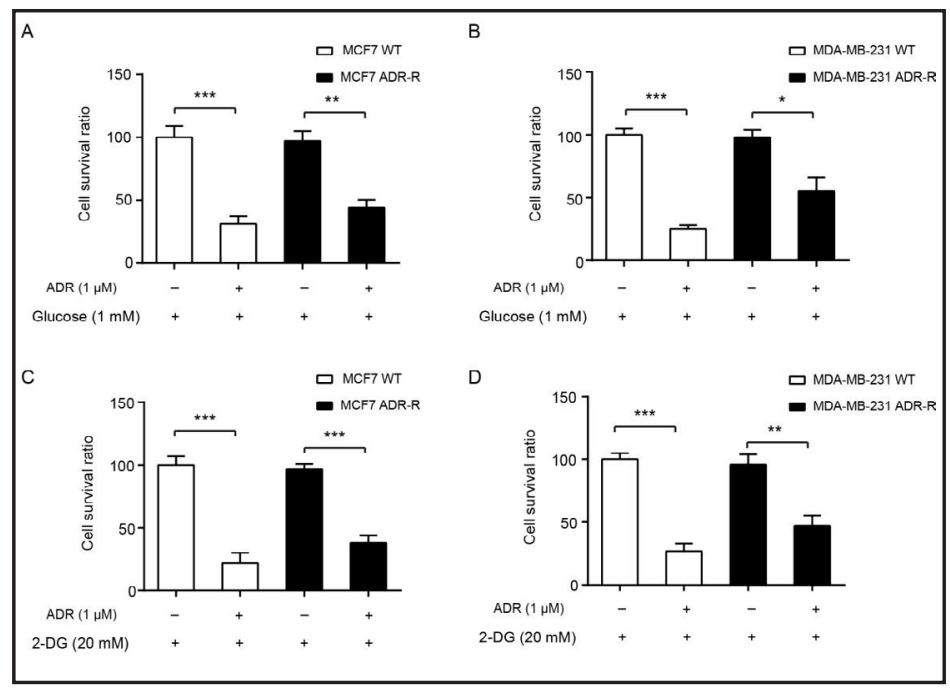

sis with 2-deoxy-D-glucose (2-DG, a classical glycolysis inhibitor) in ADR-R cells. The ADR-R cells were more sensitive to ADR in the presence of 2-DG (Fig. 2C, 2D). These data suggest that the chemoresistant phenotype of ADR cells primarily depends on enhanced glucose metabolism.

FGFR4 is the master regulator of enhanced glucose metabolism in ADR-resistant breast cancer cells

To reveal the molecular mechanism underlying the enhanced glucose metabolism in ADR-R cells, we examined RNA extracted from MCF7 and MCF7/ADR cells. The 10 genes showing the most up-regulation (ADR versus WT), including TFDP3, LOXL2, AQP3, OCT4, ABCC5, PMP22, PAK1, HPIP, FGFR4, and NRF2, are shown in Fig. 3A. Next, using real-time PCR analysis, we observed similar changes of these differentially expressed genes (Fig. 3B). Specifically, FGFR4 had the greatest change in both MCF7/ADR and MDA-MB-231/ ADR cells. Moreover, to demonstrate whether FGFR4 is responsible for ADR resistance and correlated with glucose metabolism, we genetically silenced FGFR4 in MCF7/ADR and MDA- 


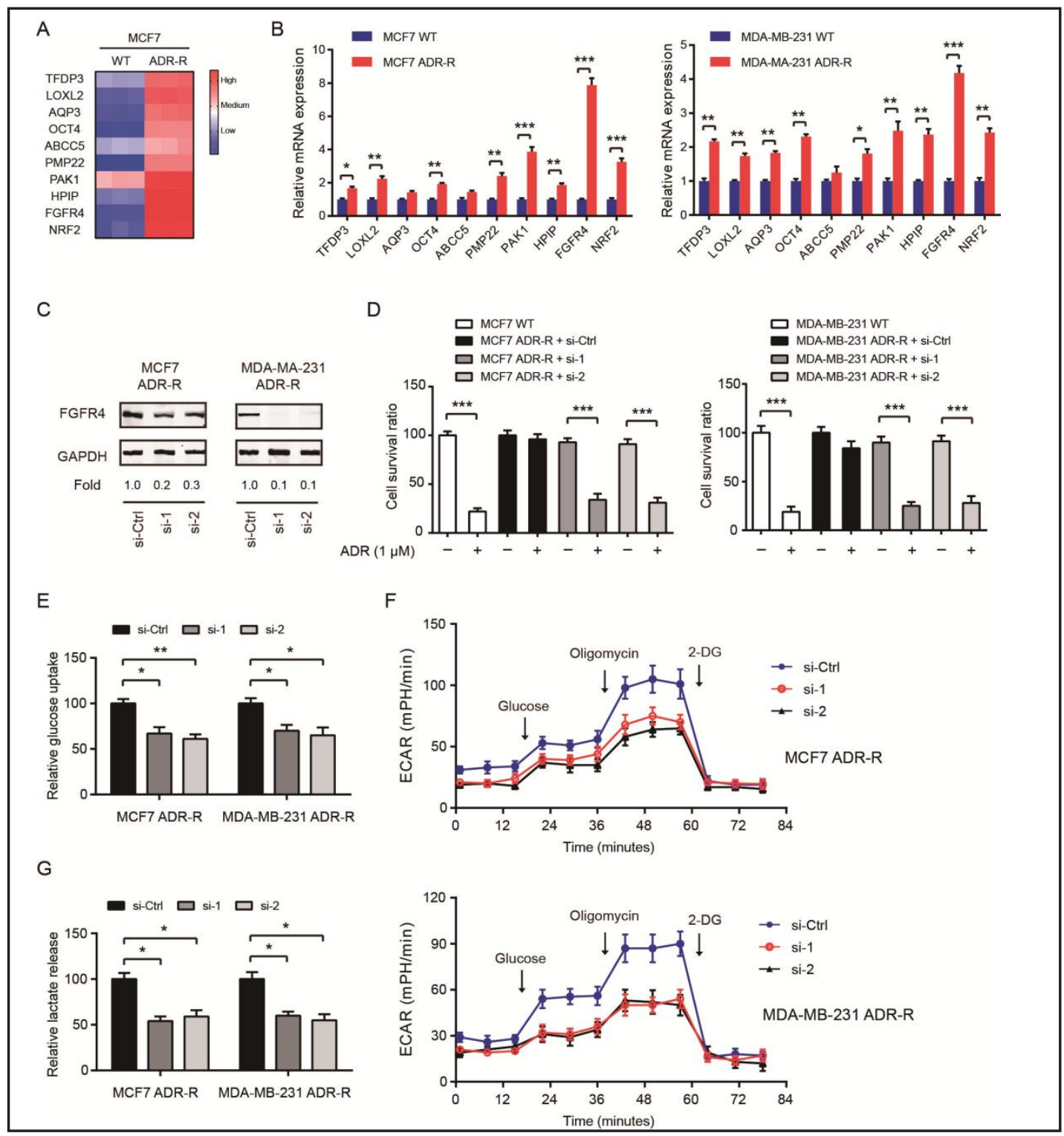

Fig. 3. FGFR4 is the master regulator of enhanced glucose metabolism in ADR-resistant breast cancer cells. (A) Genechip analysis of significantly up-regulated genes in MCF7 ADR-R cells compared with the parental WT cells. (B) Real-time qPCR analysis for significantly up-regulated genes observed in the genechip analysis. (C) The interference efficiency of FGFR4 in MCF7 ADR-R and MDA-MB-231 ADR-R cells. (D)The relative chemosensitivity of ADR-R cells after an FGFR4 knockdown. Cells were treated with $1 \mu \mathrm{M}$ ADR for $48 \mathrm{hr}$, and their survival was assessed by CCK-8 analyses. (E-G) The effect of an FGFR4 knockdown on the relative glucose consumption (E), lactate production (F), and ECAR (G)of MCF7 ADR-R and MDA-MB-231 ADR-R cells, ${ }^{*} \mathrm{p}<0.05 ;{ }^{* *} \mathrm{p}<0.01 ;{ }^{* * *} \mathrm{p}<0.001$.

MB-231/ADR cells. Two specific siRNAs targeting FGFR4 resulted in a pronounced decrease of the FGFR4 protein levels (Fig. 3C). ADR-R cells failed to respond to ADR treatment but demonstrated significantly diminished survival when FGFR4 was inhibited (Fig. 3D). The FGFR4 knockdown also significantly suppressed the glycolytic phenotypes of MCF7/ADR and MDA-MB-231/ADR cells, as demonstrated by decreased glucose uptake (Fig. 3E), reduced lactate release (Fig. 3F), and attenuated ECAR (Fig. 3G). Collectively, these results indicate that FGFR4 contributes to the ADR resistant phenotype and the enhanced glycolytic ability of ADR-R cells.

\section{KARGER}




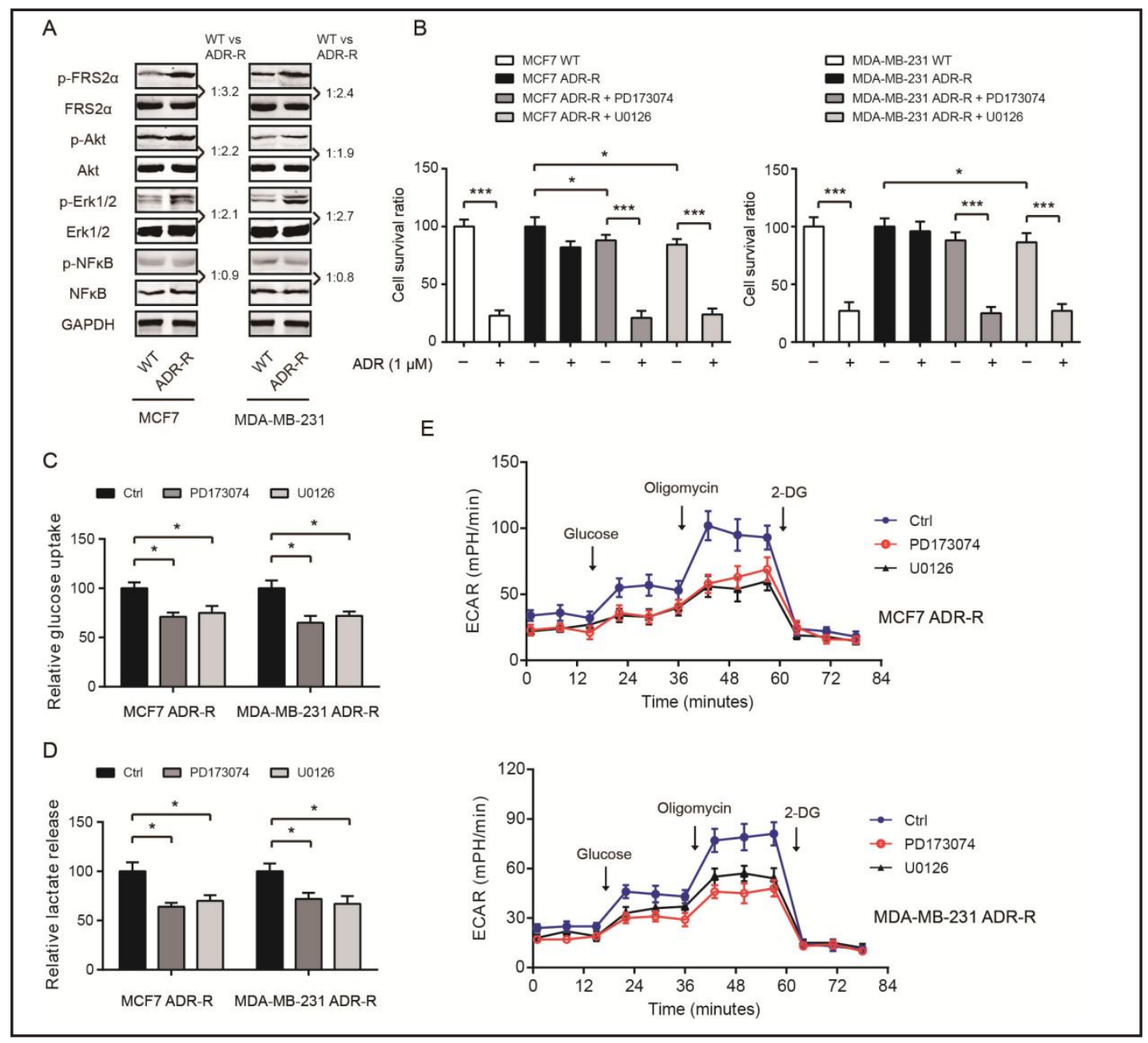

Fig. 4. Pharmacological inhibition of the FGFR4-Erk1/2 pathway increases chemosensitivity and suppresses enhanced glucose metabolism. (A) ADR-R cells and the parental WT cells were lysed and analyzed by Western blots using specific antibodies against the downstream molecules of FGFR4 signaling. The ratio of phosphorylated protein and total protein in WT and ADR-R cells is shown in the right panel.(B) The relative chemosensitivity of ADR-R cells in the presence of the FGFR4 inhibitor PD173074 (5 nM) or the Erk1/2 inhibitor U0126 (10 $\mu \mathrm{M})$. Cells were treated with $1 \mu \mathrm{M}$ ADR for $48 \mathrm{hr}$, and analyzed with CCK-8. (C-E) The relative glucose consumption (C), lactate production (D), and ECAR (E) in MCF7 ADR-R and MDA-MB-231 ADR-R cells in the presence of the FGFR4 inhibitor PD173074 or the Erk1/2 inhibitor U0126. ${ }^{*} \mathrm{p}<0.05$; $* * * \mathrm{p}<0.001$.

Pharmacological inhibition of the FGFR4-Erk1/2 pathway increases chemosensitivity and suppresses enhanced glucose metabolism

The binding of FGFR4s to the corresponding FGF ligands causes receptor dimerization and transphosphorylation of FGFR tyrosine domains [21-23]. Activated FGFR phosphorylates FGF receptor substrate 2 (FRS2) and leads to the activation of downstream signaling pathways, including MAPK, PI3K/AKT, and NF- $\mathrm{B}[15,18,24]$. As shown in Fig. 4A, we observedincreasedphospho-FRS2 and phospho-Erk1/2 levels in ADR-R cells compared with the WT cells. However, Akt and NF- $\mathrm{kB}$ phosphorylation were not significantly changed, suggesting the potential role of the FGFR4-FRS2-Erk1/2 signaling pathway in ADR resistance and glucose metabolism. To test this hypothesis, two classical pharmacological inhibitors targeting FGFR4 and Erk1/2, namely PD173074 and U0126, were used [25]. Indeed, treatment with PD173074 or U0126 significantly improved the response of ADR-R 
$\mathrm{Xu}$ et al.: FGFR4 Correlates the Glycolytic and Chemoresistant Phenotypes in Breast Cancer

cells to ADR treatment (Fig. 4B). PD173074 and U0126 also blocked the enhanced glycolytic phenotype of ADR-R cells, as revealed by glucose uptake (Fig. 4C), lactate release (Fig. 4D), and ECAR (Fig. 4E).

\section{Discussion}

Drug resistance is the major cause of the failure of clinical chemotherapy. ADR is an effective and frequently used chemotherapeutic agent for various malignancies, including breast cancer. However, the response of breast cancer patients to ADR is very poor, with no significant increase in patient survival. Previously, numerous mechanisms of ADR resistance, especially the rate of drug influx or efflux, have been identified [26-28]. In this study, we demonstrate a novel mechanism of chemoresistance by which cancer cells increase glycolytic flux.

Tumor cells can constantly and rapidly adapt to changes in the tumor microenvironment, including alterations in the levels of glucose, oxygen and key transition metals. Increased glycolytic flux has been demonstrated in many types of cancers and is believed to originate as an adaptive response to the selective pressure of the tumor microenvironment [29]. Accumulating evidence demonstrates that the compound 2-DG enhances the effect of chemotherapy against cancer cells both in vitro and in vivo [30]. We therefore hypothesized that cancer cells might depend on the glycolytic pathway for the acquisition of drug resistance and that targeting this phenotype could be exploited for therapeutic intervention. In this study, we found that ADR-R cells have increased glycolytic flux compared with their parental WT cells. Consistent with our observation, Shukla et al. demonstrated that increased glucose metabolism fuels gemcitabine resistance in Gem-resistantpancreatic cancer cells [31]. They showed that increased glycolysis led to glucose dependence and a corresponding elevation in pyrimidine biosynthesis, which increased the intrinsic levels of deoxycytidine triphosphate (dCTP). Subsequently, the increased dCTP levels diminished the effective levels of gemcitabine through molecular competition in pancreatic cancer. In addition, targeting glycolytic enzymes can also effectively enhance chemosensitivity in chemoresistant cancer cells [29, 32-34]. These effects are primarily associated with reduced glycolysis. In addition to allowing abundant cellular buildings, enhanced glycolysis can increase the intracellular ATP levels, which are crucial for ATP-dependent transporters, including P-glycoprotein (MDR1 protein), a major cause of multidrug resistance. Therefore, our data and data from other groups suggest that a combination of a glycolytic inhibitor with conventional chemotherapeutic drugs might improve the clinical effectiveness of chemotherapy.

Therefore, a combination of a glycolytic inhibitor with conventional chemotherapeutic drugs might effectively enhance chemosensitivity in chemoresistant pancreatic cancer cells and help prevent recurrence and metastasis after chemotherapy.

Next, we found that the resistance of ADR-R cells to doxorubicin correlated with FGFR4 up-regulation. Up-regulation of FGFR4 is associated with multiple oncogenic functions in cancer, including cell survival, proliferation, apoptosis, migration, and chemoresistance [14, 18].A role for FGFR4 in chemoresistance has recently been described for hepatocellular carcinoma and colorectal cancer [35, 36]. Here, using a loss-of-function study, we showed that FGFR4 knockdown improves the chemotherapy response of ADR-R cells to ADR treatment and suppresses glycolytic flux. Consistent with our observation, Roidl et al. showed that FGFR4 gene expression is up-regulated in doxorubicin-treated, apoptosis resistant cancer cell clones [37].Activating FGFR4 can inhibit the MAPK, PI3K/Akt or NF$\kappa B$ signaling pathway depending on the cellular context $[21,24]$. In this study, we observed drastic changes of Erk1/2 phosphorylation in ADR-R cells. Consistent with our observation, Roidl et al. also showed that small interfering RNA mediated knockdown of FGFR4 expression caused a decrease in Erk1/2 phosphorylation [37], suggesting that FGFR4-Erk1/2 signaling is essential for the chemoresistant phenotype of breast cancer cells. Finally, pharmacological inhibition of FGFR4 signaling by PD173074 suppressed the resistance of cancer cells to ADR treatment. PD173074 is a first-generation inhibitor specific for FGFR [38]. Therefore, 


\section{Cellular Physiology Cell Physiol Biochem 2018:47:151-160

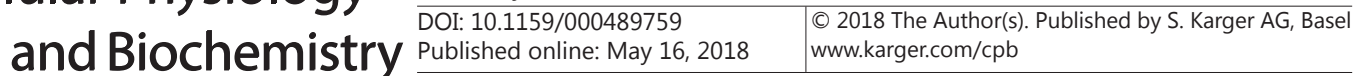 Xu et al.: FGFR4 Correlates the Glycolytic and Chemoresistant Phenotypes in Breast Cancer}

our study provides a critical rationale for targeting FGFR4 signaling in patients with ADRresistant breast cancer.

\section{Conclusion}

Our results indicate that ADR resistant breast cancer cells display enhanced glucose metabolism, thereby revealing a prevalent metabolic mechanism of ADR resistance. Targeting the driver FGFR4-Erk1/2 signaling might overcome ADR resistance in breast cancer.

\section{Acknowledgements}

This work was supported by grants from Program for Key Medical Subject of Lishui Committee of Science and Technology (Grant No. 2016zdxk01), Program for Science and Technology Department of Zhejiang Province (Grant No. 2017C33216), and Program for Health and Family Planning Commission of Zhejiang Province (Grant No. 2016152978).

\section{Disclosure Statement}

The authors confirm that there are no conflicts of interest.

\section{References}

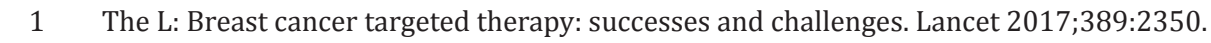

2 Harbeck N, Gnant M: Breast cancer. Lancet 2017;389:1134-1150.

- 3 Prados J, Melguizo C, Ortiz R, Velez C, Alvarez PJ, Arias JL, Ruíz MA, Gallardo V, Aranega A: Doxorubicinloaded nanoparticles: new advances in breast cancer therapy. Anticancer Agents Med Chem 2012;12:10581070 .

4 Tavangar F, Sepehri H, Saghaeian Jazi M, Asadi J: Amphotericin B potentiates the anticancer activity of doxorubicin on the MCF-7 breast cancer cells. J Chem Biol 2017;10:143-150.

-5 Siddharth S, Nayak A, Nayak D, Bindhani BK, Kundu CN: Chitosan-Dextran sulfate coated doxorubicin loaded PLGA-PVA-nanoparticles caused apoptosis in doxorubicin resistance breast cancer cells through induction of DNA damage. Sci Rep 2017;7:2143.

6 Liberti MV, Locasale JW: The Warburg Effect: How Does it Benefit Cancer Cells? Trends Biochem Sci 2016;41:211-218.

7 Dang CV. Links between metabolism and cancer: Genes Dev 2012;26:877-890.

8 Hanahan D, Weinberg RA: Hallmarks of cancer: the next generation. Cell 2011;144:646-674.

-9 Cantor JR, Sabatini DM: Cancer cell metabolism: one hallmark, many faces. Cancer discov 2012;2:881-898.

10 Lee N, Kim D: Cancer Metabolism: Fueling More than Just Growth. Mol Cells 2016;39:847-854.

11 Weber GF: Time and Circumstances: Cancer Cell Metabolism at Various Stages of Disease Progression. Front Oncol 2016;6:257.

-12 Tiong KH, Mah LY, Leong CO: Functional roles of fibroblast growth factor receptors (FGFRs) signaling in human cancers. Apoptosis 2013;18:1447-1468.

13 Eswarakumar VP, Lax I, Schlessinger J: Cellular signaling by fibroblast growth factor receptors. Cytokine Growth Factor Rev 2005;16:139-149.

14 Touat M, Ileana E, Postel-Vinay S, Andre F, Soria JC: Targeting FGFR Signaling in Cancer. Clin Cancer Res 2015;21:2684-2694.

15 Katoh M, Nakagama H: FGF receptors: cancer biology and therapeutics. Med Res Rev 2014;34:280-300.

16 Tiong KH, Tan BS, Choo HL, Chung FF, Hii LW, Tan SH, Khor NT, Wong SF, See SJ, Tan YF, Rosli R, Cheong SK, Leong CO: Fibroblast growth factor receptor 4 (FGFR4) and fibroblast growth factor 19 (FGF19) autocrine enhance breast cancer cells survival. Oncotarget 2016;7:57633-57650.

17 Pelaez-Garcia A, Barderas R, Torres S, Hernandez-Varas P, Teixido J, Bonilla F, de Herreros AG, Casal JI: FGFR4 role in epithelial-mesenchymal transition and its therapeutic value in colorectal cancer. PloS One 2013;8:e63695. 


\section{Cellular Physiology Cell Physiol Biochem 2018;47:151-160

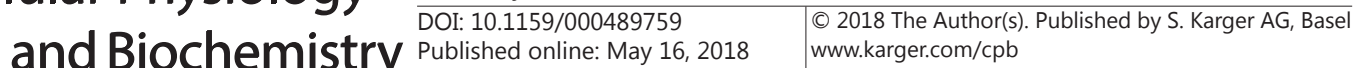 and Biochemistry}

$\mathrm{Xu}$ et al.: FGFR4 Correlates the Glycolytic and Chemoresistant Phenotypes in Breast Cancer

-18 Zaid TM, Yeung TL, Thompson MS, Leung CS, Harding T, Co NN, Schmandt RS, Kwan SY, Rodriguez-Aguay C, Lopez-Berestein G, Sood AK, Wong KK, Birrer MJ, Mok SC: Identification of FGFR4 as a potential therapeutic target for advanced-stage, high-grade serous ovarian cancer. Clin Cancer Res 2013;19:809-820.

-19 Marme F, Hielscher T, Hug S, Bondong S, Zeillinger R, Castillo-Tong DC, Sehouli J, Braicu I, Vergote I, Isabella C, Mahner S, Ferschke I, Rom J, Sohn C, Schneeweiss A, Altevogt P: Fibroblast growth factor receptor 4 gene (FGFR4) 388Arg allele predicts prolonged survival and platinum sensitivity in advanced ovarian cancer. Int J Cancer 2012;131:E586-591.

-20 Shah RN, Ibbitt JC, Alitalo K, Hurst HC: FGFR4 overexpression in pancreatic cancer is mediated by an intronic enhancer activated by HNF1alpha. Oncogene 2002;21:8251-8261.

-21 Wang S, Ding Z: Fibroblast growth factor receptors in breast cancer. Tumour Biol 2017;39:1010428317698370 Tenhagen M, van Diest PJ, Ivanova IA, van der Wall E, van der Groep P: Fibroblast growth factor receptors in breast cancer: expression, downstream effects, and possible drug targets. Endocr Relat Cancer 2012;19:R115-129.

-23 Hynes NE, Dey JH: Potential for targeting the fibroblast growth factor receptors in breast cancer. Cancer Res 2010;70:5199-5202.

24 Guo Y, Ding Y, Zhang T, An H: Sinapine reverses multi-drug resistance in MCF-7/dox cancer cells by downregulating FGFR4/FRS2alpha-ERK1/2 pathway-mediated NF-kappaB activation. Phytomedicine 2016;23:267-273.

-25 Criscitiello C, Esposito A, De Placido S, Curigliano G: Targeting fibroblast growth factor receptor pathway in breast cancer. Curr Opin Oncol 2015;27:452-456.

-26 Cao Z, Liang N, Yang H, Li S: Visfatin mediates doxorubicin resistance in human non-small-cell lung cancer via Akt-mediated up-regulation of ABCC1 Cell Prolif 2017;50:1-8.

-27 Yuan ZT, Shi XJ, Yuan YX, Qiu YY, Zou Y, Liu C, Yu H, He X, Xu K, Yin PH: Bufalin reverses ABCB1-mediated drug resistance in colorectal cancer. Oncotarget 2017;8:48012-48026.

-28 Huang H, Zhang X, Huang Z, Zhang Y, Zhou Z: Geniposide reverses multidrug resistance in vitro and in vivo by inhibiting the efflux function and expression of P-glycoprotein. Exp Ther Med 2017;13:437-442.

29 Loar P, Wahl H, Kshirsagar M, Gossner G, Griffith K, Liu JR: Inhibition of glycolysis enhances cisplatin-induced apoptosis in ovarian cancer cells. Am J Obstet Gynecol 2010;202:371 e371-378.

30 Park GB, Chung YH, Kim D: 2-Deoxy-D-glucose suppresses the migration and reverses the drug resistance of colon cancer cells through ADAM expression regulation. Anticancer Drugs 2017;28:410-420.

-31 Shukla SK, Purohit V, Mehla K, Gunda V, Chaika NV, Vernucci E, King RJ, Abrego J, Goode GD, Dasgupta A, Illies AL, Gebregiworgis T, Dai B, Augustine JJ, Murthy D, Attri KS, Mashadova O, Grandgenett PM, Powers R, Ly QP, Lazenby AJ, Grem JL, Yu F, Matés JM, Asara JM, Kim JW, Hankins JH, Weekes C, Hollingsworth MA, Serkova NJ, Sasson AR, Fleming JB, Oliveto JM, Lyssiotis CA, Cantley LC, Berim L, Singh PK: MUC1 and HIF-1alpha Signaling Crosstalk Induces Anabolic Glucose Metabolism to Impart Gemcitabine Resistance to Pancreatic Cancer. Cancer Cell 2017;32:71-87 e77.

-32 Zhao H, Duan Q, Zhang Z, Li H, Wu H, Shen Q, Wang C, Yin T: Up-regulation of glycolysis promotes the stemness and EMT phenotypes in gemcitabine-resistant pancreatic cancer cells. J Cell Mol Med 2017;21:2055-2067.

-33 Taniguchi K, Sakai M, Sugito N, Kuranaga Y, Kumazaki M, Shinohara H, Ueda H, Futamura M, Yoshida K, Uchiyama K, Akao Y: PKM1 is involved in resistance to anti-cancer drugs. Biochem Biophys Res Commun 2016;473:174-180.

34 Lepleux C, Abeilard-Lemoisson E, Duval M, Icard P, Lincet H: siPGK1 sensitizes chemoresistant human ovarian cancer cell lines to cisplatin. Anticancer Res 2012;32:4277-4286.

35 Gao L, Wang X, Tang Y, Huang S, Hu CA, Teng Y: FGF19/FGFR4 signaling contributes to the resistance of hepatocellular carcinoma to sorafenib. J Exp Clin Cancer Res 2017;36:8.

-36 Turkington RC, Longley DB, Allen WL, Stevenson L, McLaughlin K, Dunne PD, Blayney JK, Salto-Tellez M, Van Schaeybroeck S, Johnston PG: Fibroblast growth factor receptor 4 (FGFR4): a targetable regulator of drug resistance in colorectal cancer. Cell Death Dis 2014;5:e1046.

-37 Roidl A, Berger HJ, Kumar S, Bange J, Knyazev P, Ullrich A: Resistance to chemotherapy is associated with fibroblast growth factor receptor 4 up-regulation. Clin Cancer Res 2009;15:2058-2066.

-38 Dimitroff CJ, Klohs W, Sharma A, Pera P, Driscoll D, Veith J, Steinkampf R, Schroeder M, Klutchko S, Sumlin A, Henderson B, Dougherty TJ, Bernacki RJ: Anti-angiogenic activity of selected receptor tyrosine kinase inhibitors, PD166285 and PD173074: implications for combination treatment with photodynamic therapy. Invest New Drugs 1999;17:121-135. 